\title{
Design of four axis aircraft based on Android control
}

\author{
Linglong Tan ,Qing Wang , Zexin Li
}

Electronic communication engineering college, Anhui Xinhua University, Anhui Hefei, Hefei,China

\begin{abstract}
A four axle aircraft is designed with the open source firmware MWC is used as the flight control board .The MPU6050 gyro sensor and BMP085 pressure sensor are used to acquire the attitude data of aircraft in real time. Combining the Calman filtering and mean filtering, the attitude data is filtered and fused to obtain more accurate attitude information. In the control algorithm, the PID control algorithm is adopted to control the speed of the motor, which ensures that the aircraft can quickly and stably change the attitude of the flight. The Android handset is connected to an aircraft by using the WiFi signal which is launched on the four axle aircraft. The user can control the aircraft by using the appropriate App. Experimental results show that: The four axle aircraft can realize the functions of attitude, heading and hover under the control of App, and the system has friendly interface, flexible operation, stable and reliable.
\end{abstract}

\section{Introduction}

The four axis aircraft is becoming more and more popular. Its common control is to use radio remote control [1].There are three main types of remote control transmitters for four axis aircraft at present: The first one is a portable lever type remote control transmitter, the second one is a hand-held gun remote control transmitter, and another one is a small hand-held remote control transmitter for cassette keys. Gun type transmitter is the most popular remote control system at present, The gun type transmitter is a runner and a similar trigger lever to control the direction and speed respectively, remote control system of switch type analog circuit, the general model of ordinary toy remote control will use the remote control system, operation instruction is relatively simple, only the "on" and "off" of the two states, Performance and remote control distance is low. The four axis aircraft is controlled by Android phones. Using Android mobile phones to control four axis aircraft removes the traditional handheld wireless remote controller and the operation is simple.

\section{Introduction of four axis aircraft}

Four axis aircraft using MWC open source firmware for flight control board, and has a digital pressure sensor BMP085, MPU6050 digital three axis accelerometer and three axis gyro sensors and HMC5883L three axis digital magnetoresistive sensor, can help determine the four axis aircraft flying state with the sensors, and can adjust the flight attitude according to the parameters of each sensor. Therefore, the open source firmware is suitable for the development of four axis aircraft. In addition, the firmware has reserved other interface pins, such as I2C interface and serial port interface, which makes the ports for other sensors to be added later. The four axis flight control module consists of the following components:

1. master chip

Master chip is used ATmega328P microcontroller, with $1 \mathrm{~Kb}$ EEPROM and $32 \mathrm{~Kb}$ Flash, clock frequency is $16 \mathrm{MHz}$. Arduino IDE can be used to edit and burn programs [2].

\section{MPU6050 sensor}

MPU6050 integrates 3 axis gyroscope and 3 axis accelerometer, and an extensible digital motion processor Digital Motion Processor (DMP), digital sensor and connected to a third party through the $\mathrm{I} 2 \mathrm{C}$ interface. After expansion, the MPU6050 can output a 9 axis digital signal with its I2C or SPI interface. The MPU6050 sensor uses three 16 bit ADC for gyroscopes and accelerometers to convert the analog measurements into digital quantities. In use, in order to be able to accurately track the equipment fast and slow motion, the measuring range can be controlled, the range is +250 , gyroscope can measure the +500 , + $1000,+2000 \mathrm{DEG} / \mathrm{sec}$, the scope for accelerometer can be measured as $+2,+4,+8,+16 \mathrm{~g}$.

3. HMC5883L

Highly integrated HMC5883L module is a weak magnetic sensor chip with digital interface, which can 
be used in the field of magnetic field testing. HMC5883L has an integrated circuit of Honeywell patents, including amplifier circuits, bias calibration circuits, and automatic degaussing drives. HMC5883L uses Honeywell anisotropic magnetoresistive technology, which is characterized by high linearity, high accuracy and high sensitivity. This advantage is not realized by other sensor technologies. The sensor has a solid structure of low sensitivity to the orthogonal axis, and the direction and magnitude of the earth's magnetic field can be measured using this structure, with measurements ranging from Gauss to 8 Gauss.

\section{BMP085 sensor}

Four axis aircraft in flight, if you need to lock height, you need to use the pressure sensor, BMP085 is a low-power, high-precision pressure sensor, can be applied to the pressure measurement equipment. Its absolute accuracy at the lowest value can reach $0.03 \mathrm{hPa}$, at the same time, its power consumption is very low to $3 \mathrm{uA}$. The sensor can be mounted on the four axle to provide pressure data for the four axle to cause pressure changes due to height changes, and to transfer the data to the master chip for processing. On the package, the BMP085 features an ultra thin package of 8-pin ceramic leadless chip, which looks very delicate. Its communication mode is to transmit data by using $\mathrm{I} 2 \mathrm{C}$ bus and control chip.

\section{Introduction of control algorithms}

If a gyroscope or accelerometer is used alone to detect the attitude of the flight, it is impossible to provide reliable data for the balance of the aircraft. According to the characteristics of both, if the method used for filtering, fusion of gyroscope and accelerometer output signal to compensate for the cumulative error, gyro drift error and dynamic error of the acceleration sensor, can make the two mutual calibration, which can play the advantages, can also avoid their disadvantages, so the measurement can get a dip a better approximation, thus more accurate attitude measurement.

The design of the MPU6050 sensor output data using Calman filtering and mean filtering to filter and fusion, in order to obtain more accurate attitude information. Using the PID control algorithm to control the speed of the motor, to maintain the four axis aircraft in changing the attitude of the flight can be fast and stable.

\subsection{Calman filtering algorithm}

Calman filter[3] can measure a series of signals that do not contain noise completely, and can estimate the state of dynamic system optimally. It is an efficient recursive filter [4].

It is assumed that a linear stochastic differential equation is used to describe a discrete control process system:

$$
X(k)=A X(k-1)+B U(k)+W(k)
$$

The measured values of the system are:

$$
Z(k)=H X(k)+V(k)
$$

$X(k)$ is the system state of the $k$, and $\mathrm{u}$ is the amount of control that is added to the system at the $k$ moment. In formulas, $A$ and $B$ are intrinsic parameters of the system, and when the system is multiple modules, they are matrices. $k$ time measurement value is $z(k)$, when it is in the measurement system, parameter measurement system is $\mathrm{H}$ matrix. $\mathrm{W}$ and $\mathrm{V}$ respectively represent system processes and system measurements of noise. Assuming that the Gauss white noise does not vary with the system state, then the covariance of $W(k)$ and $V(k)$ is $\mathrm{Q}$ and $\mathrm{R}$. The Calman filter is the best processor for the process satisfying the above conditions.

First use system process model to predict the next state:

$X(k, k-1)=A X(k-1, k-1)+B U(k)$

$X(k, k-1)$ is the value predicted by the previous state. $X(k-1, k-1)$ is the optimal result of the previous state, and $U(k)$ is the control of the current state.

The covariance equation corresponding to $X(k-1, k-1)$ is:

$P(k, k-1)=A P(k-1, k-1) A^{T}+Q$

$P(k, k-1)$ is the value of the covariance of the $X(k, k-1), P(k-1, k-1)$ is the covariance of the $X(k-1, k-1)$, and $A^{T}$ is the transpose of the $A$, and the value of $\mathrm{Q}$ is the covariance of the system process. 
The current state measurement value of the system is collected, and the optimal estimation value of the current state $(\mathrm{k})$ can be obtained by fusing the predicted value and the measured value $X(k, k)$ :

$X(k, k)=X(k, k-1)+K_{g}(k)[Z(k)-H X(k, k-1)]$

The $K_{g}$ is Calman gain in the formula:

$$
K_{g}=\frac{P(k, k-1) H^{T}}{H P(k, k-1) H^{T}+R}
$$

In order to ensure that the Calman filter can continue running, the covariance of the $x(k, k)$ in the $k$ state is also updated:

$\mathrm{I}$ is the unit matrix in the formula and $\mathrm{I}=1$ is in the single model single measurement system. When the system is in the $k+1$ state, the $P(k, k)$ becomes the $P(k-1, k-1)$ at this point. In this way, we can do the regression calculation and realize the optimal estimation.

The average filter takes the average value of $\mathrm{N}$ values as the measured value, so that the measured value is close to the actual value, and can reduce the random error and weaken the influence of the external disturbance on the control process.

\subsection{PID control algorithm}

In the control of four axis aircraft, because there are many unknown disturbances and disturbances that affect the flight of an aircraft, the PID control [5] is adopted in this design.

The controller based on the ratio of deviation, integral and differential is called the PID controller. This automatic control law is widely used in the control system[6,7].

As shown in Fig.1, $r(t)$ is the system set value, $u(t)$ is the control quantity, and $c(t)$ is the actual output value

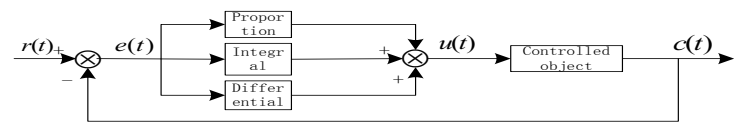

Fig. 1. Schematic diagram of PID system control

The control formula of analog PID controller is:

$$
u(t)=K_{P}\left[e(t)+\frac{1}{T_{I}} \int_{0}^{t} e(t) d t+T_{D} \frac{d e(t)}{d t}\right]
$$

In the formula, $e(t)=r(t)-c(t)$ is the system deviation; $K_{P}$ is the coefficient of proportionality;
$T_{I}$ is the integral time constant; $T_{D}$ is the differential time constant.

In the four axis flight vehicle system, the four axis can be adjusted quickly by the PID control algorithm, so that the flight parameters can be adjusted rapidly, and the stability of the four axis flight state can be maintained.

\section{Design of the system}

\section{1 overall design ideas}

The design uses Android mobile phone as the remote control, through WiFi to send control instructions to the four axis aircraft, to achieve the lifting, pitching, steering, rotating and other flying posture.After setting up the WiFi connection with the four axis, the mobile phone sends the relevant control data by judging the user gesture operation. The overall design flow chart of this system is shown in fig. 2 .

\subsection{App software design}

Open the App, first stop $2 \mathrm{~S}$ in the interface, and then enter the squared unlock page, if successful unlock mobile phone will automatically determine the current WiFi connection status, when success is connected with the four shaft, start recording connection time, while users can manipulate the two button to move on the screen, and the calculated coordinate information button control different data, sent through the network to the WiFi module on the four axis aircraft.

The flow chart of the App software design is shown in fig. 3 .

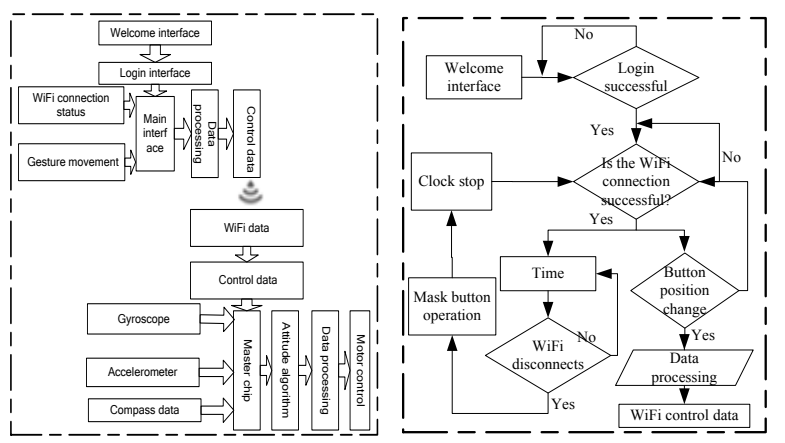

Fig.2. Overall framework of the system

Fig.3. App design flow chart 


\section{3 four axis aircraft design}

The four axis aircraft uses MWC open source firmware as the flight control board, and the master chip determines the flying posture of the aircraft through related sensors. After processing the $\mathrm{WiFi}$ data sent from the Android mobile terminal, the PPM control signal is transmitted to the master chip, and then processed into a signal to control the motor to control the attitude of the four axis flying. The design flow chart of the four axis aircraft is shown in fig. 4 .

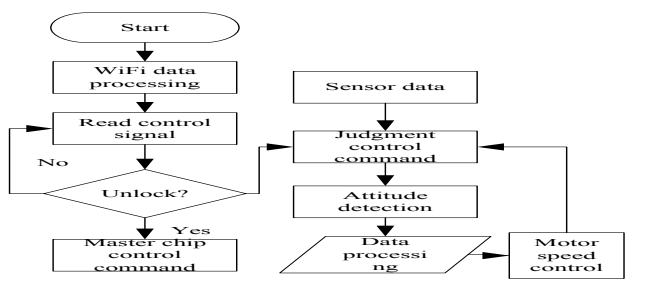

Fig.4. four axis design flow chart

\section{Design and implementation of App software}

\subsection{App main interface}

\subsection{1 layout of main interface}

The interface layout file uses the Frame Layout. Frame Layout is a layer layout, and all controls are placed in the upper left corner of the screen. The defined controls are superimposed up and down in sequence, and the controls that are placed will be displayed directly on the previous control. The previous control is blocked when the previous control size is smaller than the later control.

In the main interface, each side has a circle with a larger radius, which is the range that can be moved when the user moves the button, and the button can only move freely within the range. The upper left corner of the screen is the text displayed by the wifi connection state, and the user can see whether the current connection is wifi and the name of the connected wifi. The upper right corner of the recording connection time App and four aircraft, when disconnected, stop time, can help the user to observe the stability of the connection. There are 4 vertical bar in the middle of the screen, with the graphical representation of the current amount of rudder throttle, direction, pitch and aileron of the 4 channel value, users can easily visualize the operation of each channel.

In the main interface layout, in addition to the system controls, but also custom control, on both sides of the screen painted a radius of $350 \mathrm{px}$ circle, the circle is the range of movement of the control button. Inside the circle, two intersecting straight lines provide the button position reference function for the user. The main interface measured as shown in figure 5 .

\subsubsection{App detects the WiFi connection state program}

Detection of wifi connection state in App through the radio to listen to the wifi i connection state, when the program into the main interface, the main interface has been through the radio to listen to the wifi connection state, when the Android mobile phone connected to the wifi, the program will automatically try to connect four aircraft, and the bomb box prompts the user is working with four axis connection. Until the connection is successful, the frame closes and starts recording the connection time, then the user can manipulate the button and send a control command to the four axis aircraft. Interface measurements when connected to the WiFi are shown in fig. 6 .
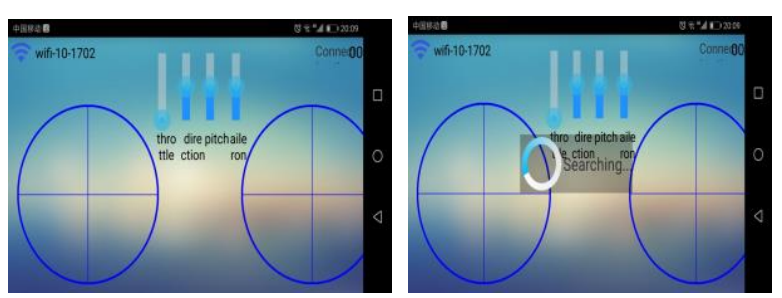

Fig.5. App master interface measurement

Fig.6. App connection four axis measurement

When the program through the radio monitoring to wifi is disconnected, the first button is set to click, is unable to send instructions to the four axis, at the same time, stop the clock connection time, and pop-up dialog box prompts the user, if you set the wifi connection. Click Cancel, the frame disappears, and click OK to jump to the system wifi settings page. When the wifi is disconnected, the main interface is shown in fig.7.

\subsubsection{App connect four axis method}

When the main interface is connected to the wifi, the method is called to open a thread, and the program has 
been in the connection loop since it came in, trying to connect to the IP address of the wifi module on the four axis aircraft. If no connection is successful within a specified time, the connection exception is indicated. If the connection is successful, you will jump out of the loop, send the connection success message to the main thread, close the connection prompt box, set the button to clickable status, and start recording the connection time. The actual measurements of the App and four axis connections are shown in fig.8.
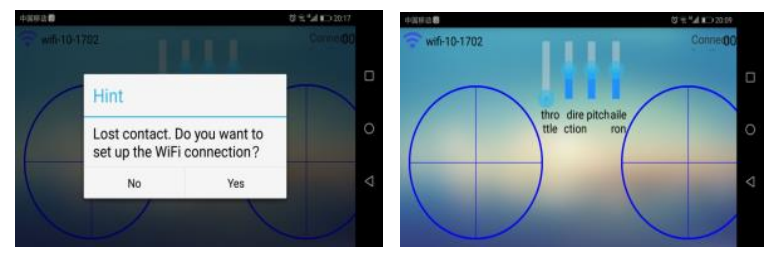

Fig.7. WiFi cut off the shot frame

Fig.8. App connection successfully measured

\subsubsection{App send and manipulate instructions}

After a successful connection, the user through the mobile button on the left and right sides to control four axis aircraft, when the user presses the button, the program will capture the current screen touch points in the $\mathrm{X}$ and $\mathrm{Y}$ axis coordinate point, when the finger moves in the circle area, the program will change $X$ and $\mathrm{Y}$ axis control with respect to the amount of acquisition and, to control set new coordinates, so that the control can be moved with the movement of the finger. In the process of moving, the program will take the measured coordinate value and coordinate value changes as parameters, calculate the control data, and then send the control data to the four axis aircraft through the network. Then, the data is received by the WiFi module on the four axis aircraft and transmitted to the master chip through the serial port.

\section{Summary}

The four axis aircraft designed in this paper is a portable aircraft with a small volume, which is powered by 4 brushless motors, with high flexibility and flexibility. The whole machine is fully electronic stabilized and easy to operate. It has a wide range of applications in many fields. Four axis aircraft flight control system based on Android design in this paper, the Android mobile phone is connected with the four axis aircraft by launching four aircraft wifi, off user with the appropriate App can control the four axis aircraft, landing and other attitude change. Not only the operation is simple, but also eliminates the traditional handheld wireless remote control, increasing the user's operating experience.

\section{ACKNOWLEDGMENT}

This work was supported by the Scientific Research Project of Anhui Xinhua College No. 2017zr003 and Natural Science Project of Anhui province No.KJ2017A618.

\section{References}

1.Zheng $\mathrm{Xu}$, Ji Zhijian, Pan Zhenzhen. Control and development of APP client for four axis aircraft based on Android [J]. industrial control computer,6(2017)

2.Yang Shubin, Huang Junrong. Design of small four axis aircraft based on real-time operating system [J]. Journal of Wuhan Institute of Technology, 1(2017)

3.Chen Peng exhibition, $\mathrm{Ru}$ Yan, Chen Xiaoyue. Simulation study on Optimization Strategy of control system of four axis aircraft $[\mathrm{J}]$. computer simulation, 12(2016)

4.Zhong Yongxi, Wu Liming, Zhang Shanshan,.WiFi system design of Micro Four Axis aircraft, [J]. single chip microcomputer and embedded system application, 11(2016)

5.Ma Zhenghua, Sun Bin. Design of short range low altitude transportation system based on four axis aircraft $[\mathrm{J}]$. computer measurement and control, 10(2016)

6.Han Ping, Sang Wilin, Shi Qing research. A new nonlinear Calman filtering method $[\mathrm{J}]$. Chinese Journal of scientific instrument, 3(2015)

7.Liu Haopeng, Long Changjiang River, Wan Peng, Wang Xiaoyi, Hu Ben. Fuzzy PID control of plant protection four axis aircraft $[\mathrm{J}]$. Chinese Journal of agricultural engineering,1(2015) 